\title{
HI properties of nearby galaxies from a volume-limited sample ${ }^{\star}$
}

\author{
I.D. Karachentsev, D.I. Makarov ${ }^{1}$, and W.K. Huchtmeier ${ }^{2}$ \\ 1 Special Astrophysical Observatory, Russian Academy of Sciences, N.Arkhyz, KChR, 357147, Russia \\ 2 Max-Planck-Institut für Radioastronomie, Auf dem Hugel 69, D-53121 Bonn, Germany
}

Received May 25; accepted July 1, 1999

\begin{abstract}
We consider global HI and optical properties of about three hundred nearby galaxies with $V_{0}<$ $500 \mathrm{~km} \mathrm{~s}^{-1}$. The majority of them have individual photometric distance estimates. The galaxy sample parameters show some known and some new correlations implying a meaningful dynamic explanation: 1) In the whole range of diameters, $1-40 \mathrm{Kpc}$, the galaxy standard diameter and rotational velocity follows a nearly linear Tully-Fisher relation, $\log A_{25} \propto(0.99 \pm 0.06) \log V_{\mathrm{m}}$. 2) The HI mass-to-luminosity ratio and the HI mass-to"total" mass (inside the standard optical diameter) ratio increase systematically from giant galaxies towards dwarfs, reaching maximum values $5 M_{\odot} / L_{\odot}$ and 3 , respectively. 3) For all the Local Volume galaxies their total mass-to-luminosity ratio lies within a range of $[0.2-16]$ $M_{\odot} / L_{\odot}$ with a median of $3.0 M_{\odot} / L_{\odot}$. The $M_{25} / L$ ratio decreases slightly from giant towards dwarf galaxies. 4) The $M_{\mathrm{HI}} / L$ and $M_{25} / L$ ratios for the sample galaxies correlate with their mean optical surface brightness, which may be caused by star formation activity in the galaxies. 5) The $M_{\mathrm{HI}} / L$ and $M_{25} / L$ ratios are practically independent of the local mass density of surrounding galaxies within the range of densities of about six orders of magnitude. 6) For the LV galaxies their HI mass and angular momentum follow a nearly linear relation: $\log M_{\mathrm{HI}} \propto(0.99 \pm 0.04) \log \left(V_{\mathrm{m}} \cdot A_{25}\right)$, expected for rotating gaseous disks being near the threshold of gravitational instability, favourable for active star formation.
\end{abstract}

Key words: galaxies: global HI parameters — galaxies

Send offprint requests to: W.K. Huchtmeier

e-mail: huchtmeier@mpifr-mpg.de

* Table in the Appendix is available only in electronic form at the CDS via anonymous ftp 130.79.128.5 or http//cdsweb.ustrasbg.fr/Abstract.html

\section{Introduction}

The acquision of global parameters of galaxies from observations in the neutral hydrogen line (Roberts 1969) revealed a new approach in investigating the evolution of gaseous and stellar subsystems of galaxies. Over the past 30 years the number of galaxies with measured HI flux and line width has been increased by two orders, reaching $N \sim 10^{4}$. Numerous publications were devoted to study relations between global $\mathrm{HI}$ and optical characteristics of galaxies. A vast comparison of HI data for about 6000 galaxies was undertaken by Roberts \& Haynes (1994). However, their sample, like is many others, limited by apparent magnitude or angular diameter of galaxies, but not by distance, which leads to a strong observational selection against intrinsically small objects.

For the analysis of global parameters of galaxies in a volume- limited sample, Huchtmeier \& Richter (1988) $(=\mathrm{HR})$ used the list of galaxies from Kraan-Korteweg \& Tammann (1979) (=KKT) with corrected radial velocities, $V_{0}<500 \mathrm{~km} \mathrm{~s}^{-1}$. The sample studied by HR contained 146 galaxies mainly of low luminosity. For the past decade the initial KKT sample has been increased almost two times in number due to the mass surveys of redshifts of galaxies from the known catalogues, revealing new nearby galaxies in the Milky Way "Zone of Avoidance", as well as special searches for dwarf galaxies in nearby groups. The increasing numbers of galaxies in the LV is mainly due to many new dwarf galaxies. It should be emphisized that the numerical growth of the KKT sample was accompanied by improving its data quality. For many faint galaxies their apparent magnitudes, angular diameters and types were specified. However, even at present one may find cases of significant discrepancy in the published data.

It should be particularly emphasized that for galaxies with $V_{0}<500 \mathrm{~km} \mathrm{~s}^{-1}$ their corrected radial velocity is a rather unreliable distance indicator because of observed deviations from the perfect cosmic Hubble flow, $V=\mathrm{HD}$ (Karachentsev \& Makarov 1996). That is why measurements of individual photometric distances for $\sim 150$ Local Volume galaxies made by different authors in the 90-ies 
allowed masses and other global parameters of nearby galaxies to be determined with a higher accuracy (see references in Karachentsev 1994, and Karachentsev \& Tikhonov 1994). The mentioned circumstances give us grounds to consider once again the relations between the global parameters of nearby galaxies based on a more complete and homogeneous sample of observational data.

\section{Initial data}

As the main source of data on optical and HI properties of galaxies, we used the last version (LEDA) of the Principal Galaxy Catalogue (Paturel et al. 1992). For many galaxies their apparent magnitudes, angular diameters and morphological types were revised using large scale CCD frames obtained with the SAO 6-meter telescope and the 2.6meter Nordic telescope. From the list of galaxies with $V_{0}<500 \mathrm{~km} \mathrm{~s}^{-1}$ we excluded about 100 objects in the central region of the Virgo cluster and also more than 50 fictitious "nearby galaxies" (i.e. globular clusters, planetary nebulae or cases when the radial velocity is inherent in a bright star projected on a distant galaxy). Our sample (the Local Volume sample $=\mathrm{LV}$ ) is updated by new objects from the list of Karachentseva \& Karachentsev (1998) and other recent sources. Particularly, there were taken into account the results of $\mathrm{HI}$ searches for nearby dwarf galaxies made by Huchtmeier et al. (1997, 1999) and Cote et al. (1997). Our updated version of the KKT sample contains a total of 303 galaxies. For the galaxies with measured HI flux, $S$ (Jansky $\mathrm{km} \mathrm{s}^{-1}$ ), corrected for beam-filling effect we calculated the mass of its neutral hydrogen

$\log \left(M_{\mathrm{HI}} / M_{\odot}\right)=\log S+2 \log D+5.37$,

where $D$ is the distance to a galaxy in Mpc. For 188 galaxies we used individual photometric distance estimates. For 47 galaxies located within the known groups we adopted the median distance of each group, and for 83 galaxies their distances were determined via radial velocity: $D=V_{0} / H$. The local value of the Hubble parameter was taken to be $H=70 \mathrm{~km} \mathrm{~s}^{-1} / \mathrm{Mpc}$. The radial velocity of galaxies, $V_{0}$, was corrected for the solar motion with respect to the Local Group centroid towards $l_{\mathrm{A}}=93^{\circ}, b_{\mathrm{A}}=-4^{\circ}$ with the $V_{\mathrm{A}}=316 \mathrm{~km} \mathrm{~s}^{-1}$ (Karachentsev \& Makarov 1996). Calculating the total blue luminosity of the galaxy, $L / L_{\odot}$, and its standard linear diameter, $A_{25}$ (in $\mathrm{Kpc}$ ), we took into account corrections for the Galactic extinction and the galaxy inclination in the manner adopted in PGC. For heavy obscured objects we used extinction values from the IR sky map by Schlegel et al. (1998). To estimate the "total" mass enclosed inside the standard angular diameter, $a_{25}$, (in arcmin) we used the relation

$\log \left(M_{25} / M_{\odot}\right)=2 \log V_{\mathrm{m}}+\log a_{25}+\log D+4.52$,

where $V_{\mathrm{m}}$ means the galaxy rotation amplitude (in $\mathrm{km} \mathrm{s}^{-1}$ ) corrected for inclination and turbulence. For dwarf galaxies inclination values are rather uncertain as they are normally derived from uncertain axial ratios. The transformation of the galaxy $\mathrm{HI}$ line width $W_{50}$ or $W_{20}$ into $V_{\mathrm{m}}$ was done following the model of corrections for turbulent motions by Tully (1988) with parameters $\sigma_{\mathrm{t}}=19 \mathrm{~km} \mathrm{~s}^{-1}$ and $\sigma_{z}=12 \mathrm{~km} \mathrm{~s}^{-1}$. Due to the uncertainty of correction for inclination of galaxies having axial ratio $b / a>0.8$ we did not determine the total mass of such galaxies.

The resulting list of basic parameters for the considered galaxies is given in the Appendix. Its columns contain: (1) the galaxy name (And III, Cas 1, etc.) or number in different catalogues and lists: $\mathrm{NGC}(\mathrm{N}), \mathrm{IC}, \mathrm{UGC}$ (U), UGCA (UA), PGC (P), DDO, KK (Karachentseva \& Karachentsev 1998), (2) its rough coordinates on (1950.0), (3) blue total magnitude, (4) corrected radial velocity, (5) morphological type, (6) distance in $\mathrm{Mpc}$ with indication of its origion: $\mathrm{P}$ - photometric, $\mathrm{M}$ - from membership, $\mathrm{H}$ from the Hubble low, (7) tidal index, (8) logarithm of corrected linear diameter in Kpc, (9) logarithm of luminosity in solar units, (10) logarithm of rotational velocity in $\mathrm{km} \mathrm{s}^{-1}$ corrected for inclination, (11) logarithm of "total" mass-to-luminosity ratio in solar units, (12) logarithm of HI mass-to-luminosity ratio in solar units, (13) logarithm of fractional HI mass, (14) mean optical surface brightness in mag/square arcsec. In total the Appendix contains data on 275 galaxies with $V_{0}<500 \mathrm{~km} \mathrm{~s}^{-1}$, and 28 dwarf spheroidal galaxies without $V_{0}$ but being highly probable members of the known nearby groups. We add to the list also 15 galaxies with $500<V_{0}<1000 \mathrm{~km} \mathrm{~s}^{-1}$ having distance estimates within $10 \mathrm{Mpc}$. References to sources of data on the presented galaxies are accessable from the e-mail address: dim@sao.ru.

\section{Correlation statistics for basic variables}

In our sample about $95 \%$ of the galaxies are of irregular and spiral type. Between their global parameters $V_{\mathrm{m}}$, $A_{25}, L_{\mathrm{B}}, M_{\mathrm{HI}}$ rather close correlations can be seen, indicating important structural and dynamic properties of dwarf and giant galaxies. In Table 1 we present numerical parameters for the linear regression, $y=k \cdot x+c$, where the variables $x$ and $y$ correspond to logarithms of different integral characteristics of the galaxies. The table columns contain: $N$ - the number of galaxies in the subsample; $r(x, y)$ - the correlation coefficient in percent, its value from Huchtmeier \& Richter (1988) is given in brackets; $\sigma(y)$ - the standard deviation with respect to the regression line; $k$ and $c$ - the regression parameters with their standard deviations.

Two upper lines in the table describe the Tully-Fisher relation for the galaxies with $V_{0}<500 \mathrm{~km} \mathrm{~s}^{-1}$. A comparison of $r(x, y)$ values for our sample with the earlier data from HR shows good agreement. The scatter of galaxies on the Tully-Fisher diagrams appears to be lower when new 
Table 1. Coefficients of linear regression, $y=k x+c$, for the global parameters of nearby galaxies

\begin{tabular}{|c|c|c|c|c|c|c|c|}
\hline$y$ & $x$ & $N$ & \multicolumn{2}{|c|}{$r(x y)$} & $\sigma(y)$ & $k$ & $c$ \\
\hline $\log \left(A_{25}\right)$ & $\log \left(V_{\mathrm{m}}\right)$ & 194 & 78 & {$[74]$} & 0.26 & $0.99 \pm 0.06$ & $-0.95 \pm 0.09$ \\
\hline $\log (L)$ & $\log \left(V_{\mathrm{m}}\right)$ & 195 & 87 & {$[83]$} & 0.48 & $2.55 \pm 0.10$ & $4.40 \pm 0.17$ \\
\hline $\log \left(A_{25}\right)$ & $\log (L)$ & 318 & 92 & [91] & 0.18 & $0.35 \pm 0.01$ & $-2.32 \pm 0.07$ \\
\hline $\log \left(V_{\mathrm{m}}\right)$ & $\log (L)$ & 195 & 87 & [83] & 0.16 & $0.30 \pm 0.01$ & $-0.92 \pm 0.10$ \\
\hline $\log \left(M_{\mathrm{H}} / L\right)$ & $\log (L)$ & 255 & -50 & {$[-51]$} & 0.46 & $-0.25 \pm 0.03$ & $1.80 \pm 0.23$ \\
\hline $\log \left(M_{25} / L\right)$ & $\log (L)$ & 194 & -2 & {$[-12]$} & 0.35 & $-0.01 \pm 0.03$ & $0.47 \pm 0.22$ \\
\hline $\log \left(M_{\mathrm{H}} / M_{25}\right)$ & $\log (L)$ & 191 & -57 & {$[-39]$} & 0.42 & $-0.30 \pm 0.03$ & $1.80 \pm 0.27$ \\
\hline $\log \left(\Sigma_{\mathrm{HI}}\right)$ & $\log (L)$ & 255 & -9 & {$[-2]$} & 0.45 & $-0.04 \pm 0.03$ & $7.2 \pm 0.2$ \\
\hline $\log \left(M_{\mathrm{H}} / L\right)$ & $\log \left(A_{25}\right)$ & 255 & -35 & {$[-31]$} & 0.50 & $-0.42 \pm 0.07$ & $-0.1 \pm 0.1$ \\
\hline $\log \left(M_{25} / L\right)$ & $\log \left(A_{25}\right)$ & 194 & 12 & [4] & 0.35 & $0.10 \pm 0.06$ & $0.35 \pm 0.04$ \\
\hline $\log \left(M_{\mathrm{H}} / M_{25}\right)$ & $\log \left(A_{25}\right)$ & 191 & -49 & {$[-34]$} & 0.45 & $-0.60 \pm 0.08$ & $-0.4 \pm 0.1$ \\
\hline $\log \left(\Sigma_{\mathrm{HI}}\right)$ & $\log \left(A_{25}\right)$ & 255 & -21 & {$[-22]$} & 0.44 & $-0.22 \pm 0.06$ & $7.0 \pm 0.1$ \\
\hline $\log \left(M_{\mathrm{H}} / L\right)$ & $\log \left(V_{\mathrm{m}}\right)$ & 191 & -41 & {$[-32]$} & 0.46 & $-0.64 \pm 0.10$ & $0.7 \pm 0.2$ \\
\hline $\log \left(M_{25} / L\right)$ & $\log \left(V_{\mathrm{m}}\right)$ & 194 & 43 & {$[38]$} & 0.32 & $0.46 \pm 0.07$ & $-0.31 \pm 0.11$ \\
\hline $\log \left(M_{\mathrm{H}} / M_{25}\right)$ & $\log \left(V_{\mathrm{m}}\right)$ & 191 & -71 & {$[-64]$} & 0.36 & $-1.10 \pm 0.08$ & $0.98 \pm 0.13$ \\
\hline $\log \left(\Sigma_{\mathrm{HI}}\right)$ & $\log \left(V_{\mathrm{m}}\right)$ & 191 & -6 & {$[12]$} & 0.41 & $-0.08 \pm 0.09$ & $7.00 \pm 0.15$ \\
\hline $\log \left(M_{\mathrm{H}} / L\right)$ & $\Sigma_{\mathrm{B}}$ & 255 & 54 & - & 0.44 & $0.30 \pm 0.03$ & $-7.4 \pm 0.7$ \\
\hline $\log \left(M_{25} / L\right)$ & $\Sigma_{\mathrm{B}}$ & 194 & 33 & - & 0.33 & $0.12 \pm 0.02$ & $-2.5 \pm 0.6$ \\
\hline $\log \left(M_{\mathrm{H}} / M_{25}\right)$ & $\Sigma_{\mathrm{B}}$ & 191 & 38 & - & 0.47 & $0.21 \pm 0.04$ & $-5.8 \pm 0.9$ \\
\hline $\log \left(M_{\mathrm{H}} / L\right)$ & Type & 255 & 60 & {$[56]$} & 0.42 & $0.10 \pm 0.01$ & $-1.12 \pm 0.07$ \\
\hline $\log \left(M_{25} / L\right)$ & Type & 194 & -10 & {$[9]$} & 0.35 & $-0.01 \pm 0.01$ & $0.51 \pm 0.07$ \\
\hline $\log \left(M_{\mathrm{H}} / M_{25}\right)$ & Туре & 191 & 71 & [63] & 0.36 & $0.12 \pm 0.01$ & $-1.76 \pm 0.08$ \\
\hline $\log \left(M_{\mathrm{H}} / L\right)$ & $\Theta$ & 255 & -11 & - & 0.52 & $-0.04 \pm 0.02$ & $-0.32 \pm 0.03$ \\
\hline $\log \left(M_{25} / L\right)$ & $\Theta$ & 194 & -4 & - & 0.35 & $-0.01 \pm 0.02$ & $0.42 \pm 0.03$ \\
\hline $\log \left(M_{\mathrm{H}}\right)$ & $\log \left(V_{\mathrm{m}} \cdot A_{25}\right)$ & 191 & 89 & - & 0.36 & $0.99 \pm 0.04$ & $5.9 \pm 0.1$ \\
\hline
\end{tabular}

photometric data and photometric distance estimates are used instead of kinematic ones, $V_{0} / H$. It should be noted that the linear diameter of a galaxy and its rotational velocity follow a linear relation with $k=0.99 \pm 0.06$ in the whole range of diameters: from $1 \mathrm{Kpc}$ to $40 \mathrm{Kpc}$. The same property was found also for thin disk-like galaxies viewed edge-on (Karachentsev et al. 1999). Apparently, the linear relation $A_{25} \propto V_{\mathrm{m}}$ has a fundamental kinematic significance, reflecting conditions of formation and equilibrium of gaseous disks of galaxies.

Due to the tight correlations between luminosity, linear diameter and rotational velocity of the galaxies each of these parameters may be considered as a suitable argument to distinguish between giant, normal and dwarf objects. However, below we give preference to $V_{\mathrm{m}}$ as a variable which is independent of distance determination errors.

Figure 1 presents the distribution of the LV galaxies according to their rotational velocity and hydrogen massto-luminosity ratio. Here both variables are independent of the galaxy distance. These data confirm the well-known fact (HR, McGaugh \& de Blok 1997) that the amount of

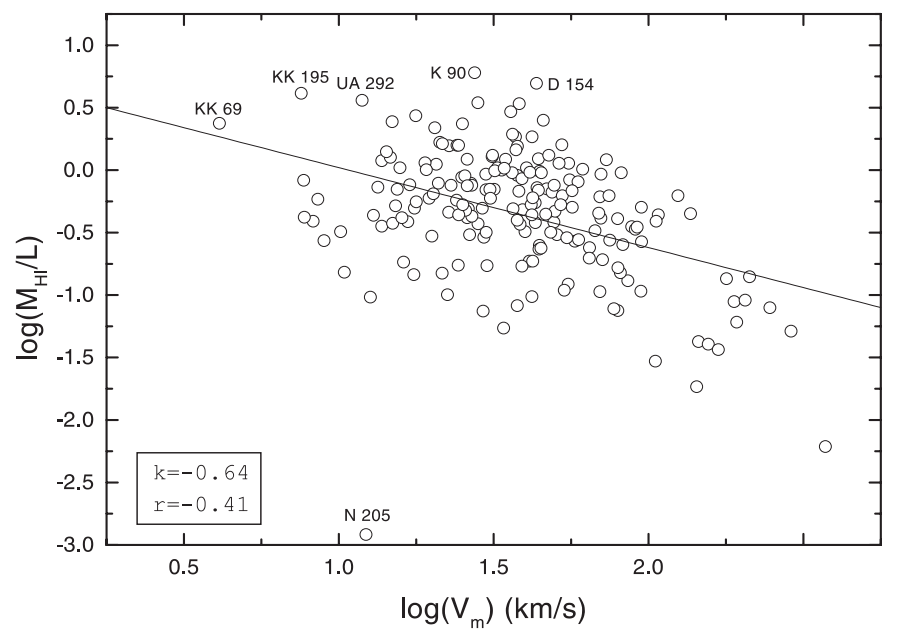

Fig. 1. The relationship between the hydrogen mass-toluminosity ratio and the rotational velocity. The solid line shows the least-squares regression. Some galaxies with extreme parameters, like NGC 205, DDO 154, UGCA 292, are indicated with their name in the figure. The quantities " $r$ " and " $k$ " in a corner correspond to the regression parameters in the Cols. (4) and (7) of Table 1 


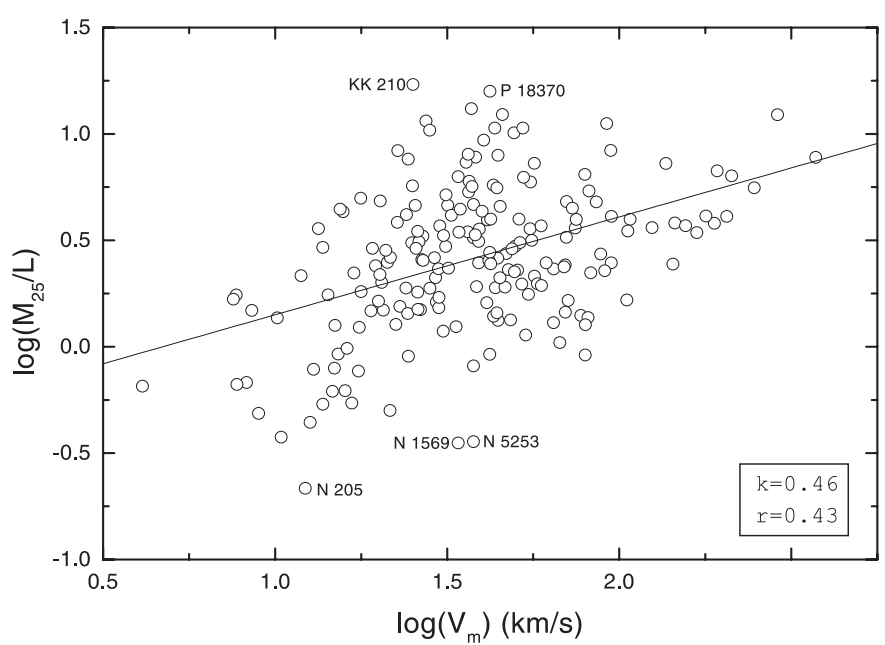

Fig. 2. The "total" mass-to-luminosity ratio versus the rotational velocity. The least-squares regression parameters, $r$ and $k$, from Table 1 are shown in a corner

hydrogen mass per unit of luminosity increases from giant spirals towards dwarfs. For some dwarf systems (K 90, DDO 154, and UGCA 292) their $M_{\mathrm{HI}} / L$ ratio reaches the maximum value, $\sim 5 M_{\odot} / L_{\odot}$. The distribution of galaxies according to the "total" mass-to-luminosity ratio and rotational velocity is given in Fig. 2. Unlike $M_{\mathrm{HI}} / L$, the $M_{25} / L$ ratio tends to decrease from giant spirals to dwarf galaxies. The same result was derived by HR (line 14 in Table 1) and Broeils \& Rhee (1997). It should be noted, however, that $M_{25} / L$ is practically independent of the galaxy luminosity (line 6 in Table 1). Moreover, some authors (HR, Salpeter \& Hoffman 1996) point even to a small increase in $M_{25} / L$ towards dwarf galaxies, which gives grounds to assume a growth of relative amount of Dark Matter towards dwarf galaxies. But the origin of this difference may simply be caused by the statistical nature of the relations: $M_{25} / L \propto V_{\mathrm{m}}$ and $M_{25} / L \propto L$, when mesurement errors of the observables have different influence on the correlation coefficients.

As it is seen in Fig. 2, the value of $M_{25} / L$ for the considered galaxies occupies a range from 0.2 to $16 M_{\odot} / L_{\odot}$ with a median of $3.0 M_{\odot} / L_{\odot}$. The minimum "total" mass-to-luminosity ratios are characteristic of galaxies having a high surface brightness with signs of active star formation (NGC 1569, NGC 5253). The maximum $M_{25} / L$ ratios are inherent in galaxies of low surface brightness like KK 210, PGC 18370, K 15 and K 90.

Staveley-Smith \& Davies (1988) and Huchtmeier \& Richter (1988) noted that the hydrogen mass-to- "total" mass ratio increases from giant towards dwarf systems. This known effect is also well seen in Fig. 3 for the sample of nearby galaxies. This relation has a clearer shape, when the rotational velocity is used as the argument instead of luminosity or linear diameter (see lines 7,11 , and 15 in Table 1). The median value of $M_{\mathrm{HI}} / M_{25}$ for the $\mathrm{LV}$ galaxies is about 0.25. Several dwarf systems like

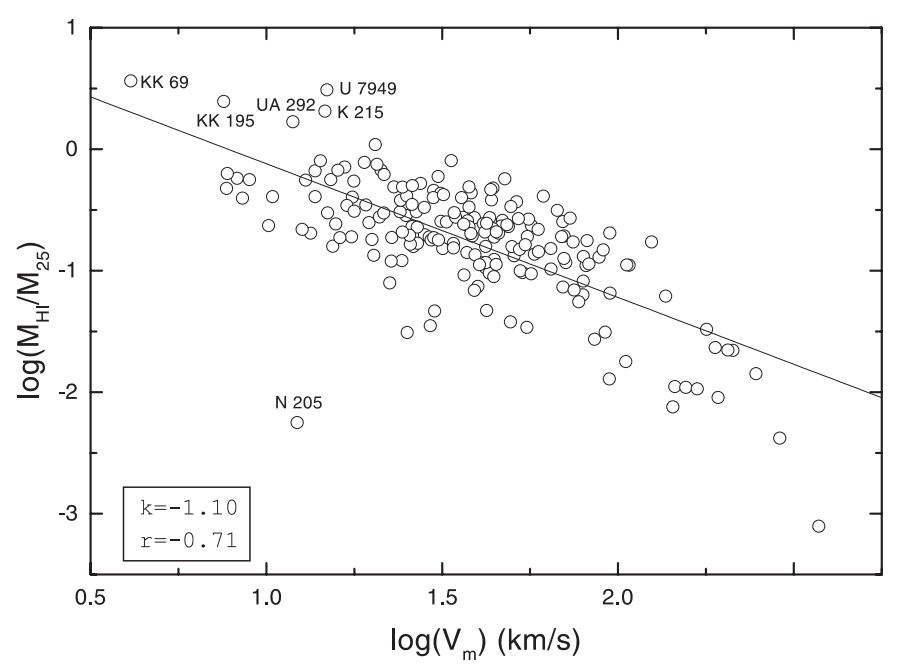

Fig. 3. The fractional HI mass shown as a function of rotational velocity. The solid line shows the least-squares regression. Some galaxies with extreme parameters, like NGC 205, DDO 154, UGCA 292, are indicated with their name in the figure. The quantities " $r$ " and " $k$ " in a corner correspond to the regression parameters in the Cols. (4) and (7) of Table 1

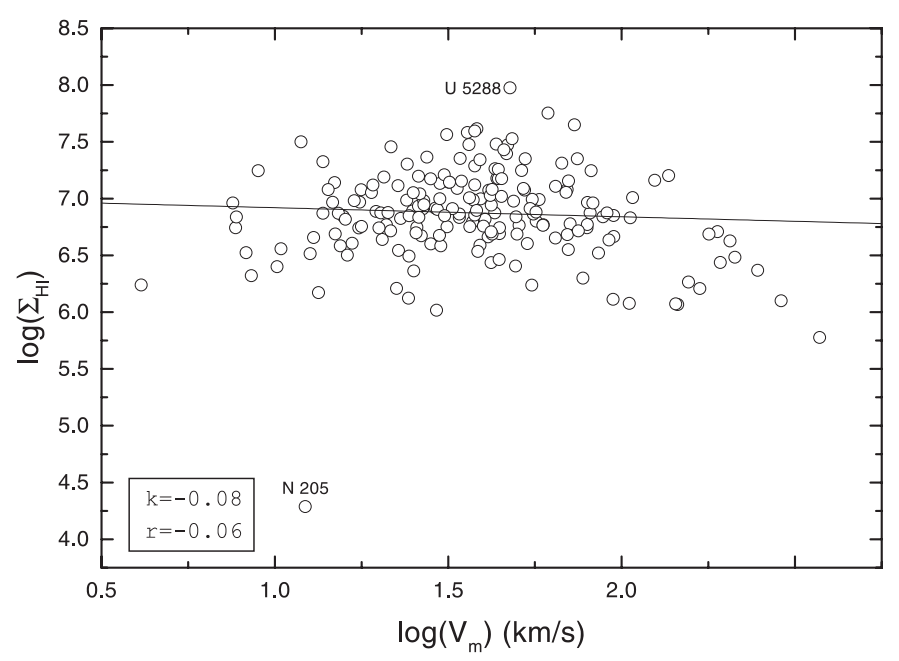

Fig. 4. The HI surface density within the standard linear diameter (in $M_{0}$ per $\mathrm{Kpc}^{2}$ ) as a function of rotational velocity. The solid line shows the least-squares regression. Some galaxies with extreme parameters, like NGC 205, are indicated with their name in the figure. The quantities " $r$ " and " $k$ " in a corner correspond to the regression parameters in the Cols. (4) and (7) of Table 1

UGC 7949, K 215, and UGCA 292 have $M_{\mathrm{HI}} / M_{25}$ in the range of $[1-3]$, which suggests that the true total mass of some dwarf galaxies exceeds their mass within $R_{25}$ at least by $(2-3)$ times in accordance with Broeils (1992). An example of such a system is DDO 154 (Carignan \& Beaulieu, 1989). But the three objects mentioned above seem to be even more HI-extended and unusual, deserving a detailed kinematic study in the HI line.

In Fig. 4 a plot of the HI surface density (in $M_{\odot}$ per $\mathrm{Kpc}^{2}$ ) versus $V_{\mathrm{m}}$ is displayed. The least-squares fit of these 


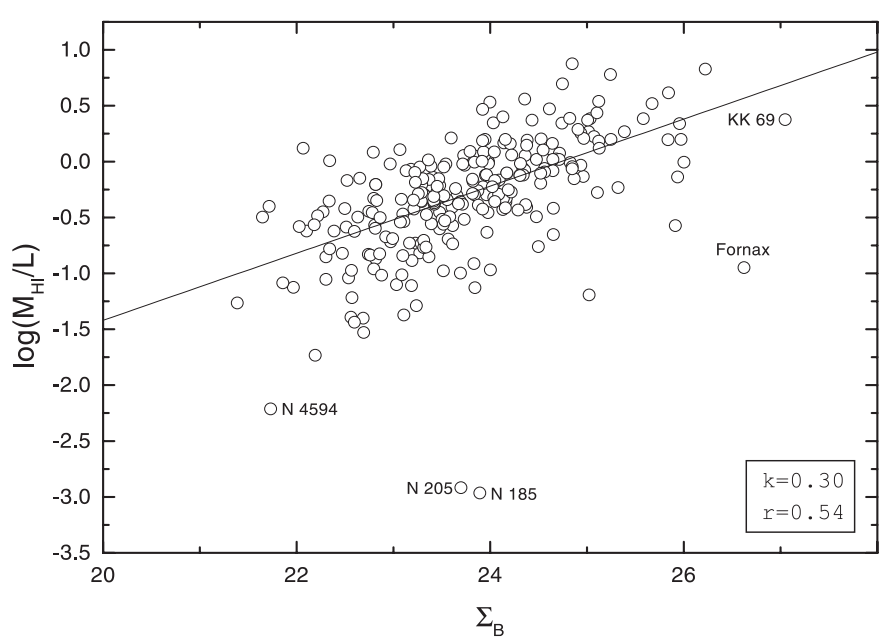

Fig. 5. The HI mass-to-blue luminosity ratio versus the mean blue surface brightness (in mag/arcsec ${ }^{2}$ ). The solid line shows the least-squares regression. Some galaxies with extreme parameters, like NGC 205, are indicated with their name in the figure. The quantities " $r$ " and " $k$ " in a corner correspond to the regression parameters in the Cols. (4) and (7) of Table 1

data, shown by the solid line, yields a slight decrease in $\Sigma_{\mathrm{HI}}$ towards giant galaxies unsignificant at the 1-sigma level.

\section{Effects of optical surface brightness and type}

Above we briefly described the relations of basic global parameters of nearby galaxies with their blue luminosity or rotational velocity. Bothun et al. (1997) and some other authors argued that galaxies of high and very low surface brightness have essentially different conditions for star formation. This must lead to a difference in their HI content and global structure. In Fig. 5 the hydrogen massto-luminosity ratio for the LV galaxies is plotted versus the mean blue surface brightness. These data show that the relative content of hydrogen drops apparently with increasing surface brightness. It may be caused by evolutionary transformation of the gaseous component of the galaxy into its stellar population (McGaugh \& de Blok 1997).

On average the hydrogen mass-to-luminosity ratio varies by a factor of $\sim 30$ with a maximum variation of the mean surface brightness by 5 magnitudes. A similar diagram for the total mass-to-luminosity ratio (Fig. 6) reveals only a slight correlation with the optical surface brightness of the galaxy.

According to Roberts \& Haynes (1994) the mean ratios, $M_{\mathrm{HI}} / L$ and $M_{\mathrm{HI}} / M_{25}$, increase smoothly from early morphological types towards late ones, but the ratio $\left\langle M_{25} / L \mid T\right\rangle$ appears to be approximately constant. In the considered volume-limited sample the early type galaxies are represented only in small numbers. Nevertheless, the regression coefficients in lines 20-22 of Table 1 agree well

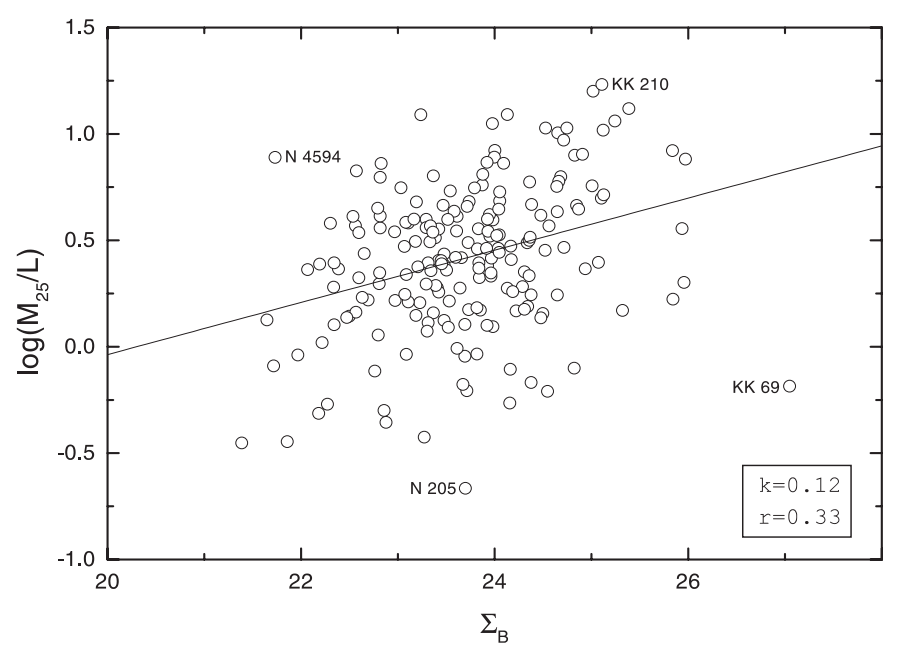

Fig. 6. The total mass-to-luminosity ratio as a function of optical surface brightness. The solid line shows the leastsquares regression. Some galaxies with extreme parameters, like NGC 205, are indicated with their name in the figure. The quantities " $r$ " and " $k$ " in a corner correspond to the regression parameters in the Cols. (4) and (7) of Table 1

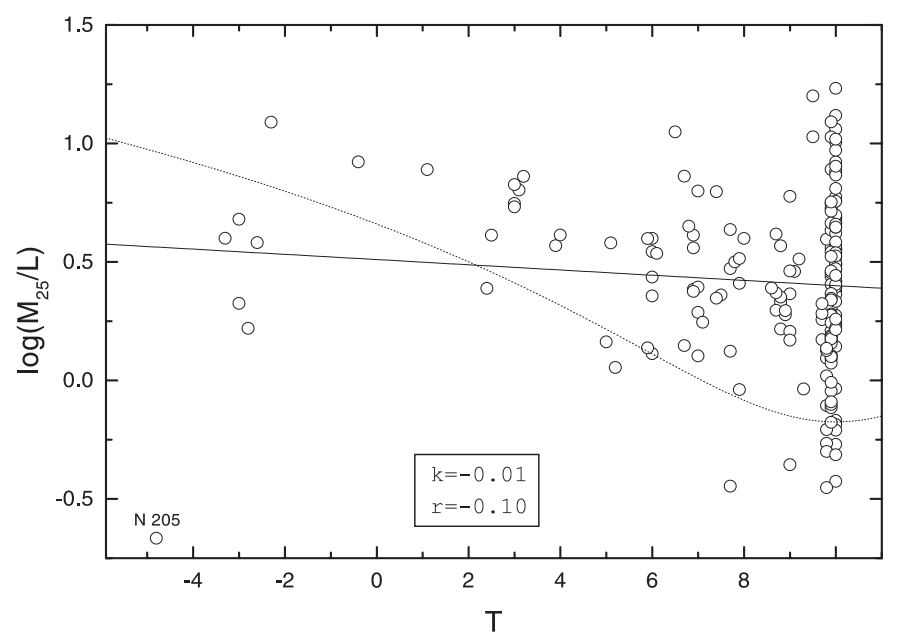

Fig. 7. The total mass-to-luminosity ratio against the morphological type. The solid line shows the least-squares regression, the dashed line is the relation for a purely stellar population of galaxies caused by their evolution according to Hoffman et al. (1996)

with the data from Roberts \& Haynes derived for the Local Supercluster sample.

In Fig. 7 a plot of the $M_{25} / L$ versus morphological type is shown for our sample, where the straight line represents the linear regression. The dashed line refers to the variation of $M_{25} / L$ along the morphological sequence for a purely stellar population of galaxies caused by their evolution (Larson \& Tinsley 1978; Hoffman et al. 1996).

\section{Environment effects}

Many publications describe a segregation of galaxies by morphological types and HI content as dependent on the 


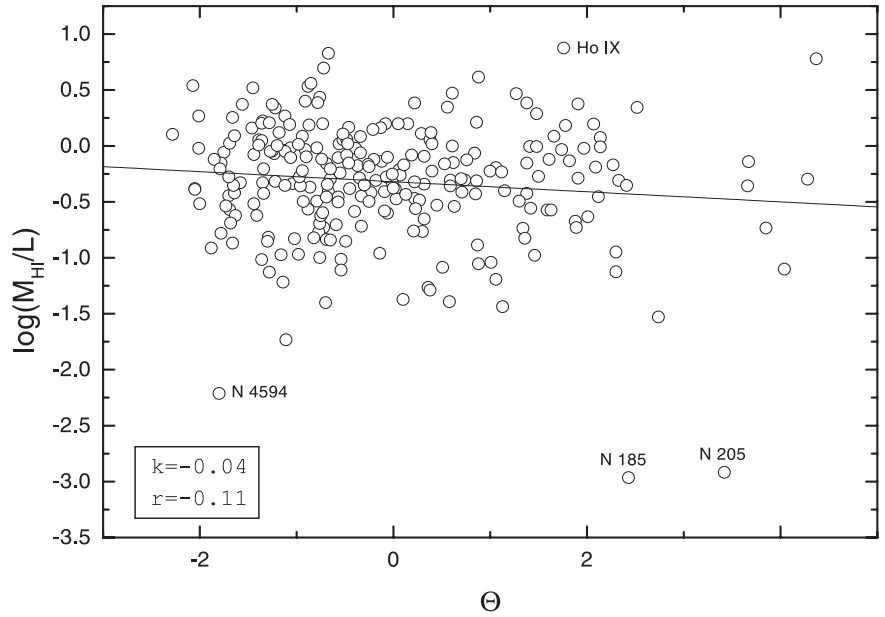

Fig. 8. The plot of HI mass-to-luminosity ratio versus the "tidal index" defined above and proportional to the local mass density. The solid line shows the least-squares regression. Some galaxies with extreme parameters, like NGC 205, are indicated with their name in the figure. The quantities " $r$ " and " $k$ " in a corner correspond to the regression parameters in the Cols. (4) and (7) of Table 1

number density of surrounding galaxies. The most distinct deficiency of HI-rich galaxies is seen in the central parts of clusters (Dressler 1984; Giovanelli \& Haynes 1991). But the data on segregation outside galaxy clusters look rather controversal.

To describe the local mass density around a galaxy " $i$ " we introduced the so-called "tidal index" or isolation index (Karachentsev \& Makarov 1998):

$\Theta_{i}=\max \left\{\log \left(M_{k} / D_{i k}^{3}\right)\right\}+C, i=1,2, \ldots N$,

where $M_{k}$ is the total mass of any neighbouring galaxy separated from the considered galaxy by a distance of $D_{i k}$. For every galaxy " $i$ " we found its "main disturber", producing the maximum density enhancement, $\Delta \rho \sim$ $M_{k} / D_{i k}^{3}$. The value of constant $C$ is choosen so that $\Theta=0$ when the Keplerian cyclic period of the galaxy with respect to its main disturber equals the cosmic Hubble time, $1 / \mathrm{H}$. Therefore, galaxies with $\Theta<0$ may be considered as well isolated objects.

The distribution of the $L V$ galaxies in $M_{\mathrm{HI}} / L$ versus index $\Theta$ is plotted in Fig. 8. As it can be seen from this diagram, in the whole range of local densities, $\Delta \log \rho_{k}=6$, the mean hydrogen mass-to-luminosity ratio remains approximately constant, showing an insignificant segregation effect from environment. It should be, however, noted that many spheroidal dwarf galaxies, whose HI-fluxes lie below a threshold detection, are not represented in Fig. 8, which produces an effect of observational selection. Another global parameter of galaxies, $M_{25} / L$, seems also to have the same mean value as for very isolated galaxies as well as for tight companions of massive galaxies (see line 24 in Table 1).

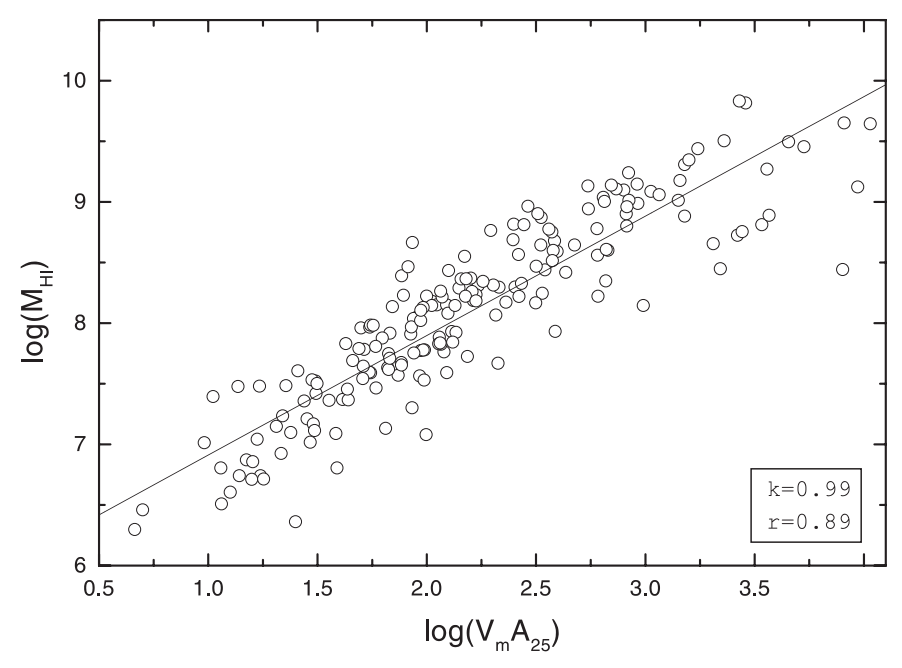

Fig. 9. The HI mass as a function of angular momentum represented by a product of rotational velocity of the galaxy on its standard linear diameter. The solid line shows the least-squares regression. The quantities " $r$ " and " $k$ " in a corner correspond to the regression parameters in the Cols. (4) and (7) of Table 1

\section{HI mass and angular momentum}

For the disks of gas-rich galaxies, where the active star formation is going on, the amount of hydrogen mass and angular momentum, proportional to $\left(V_{\mathrm{m}} \cdot A_{25}\right)$, must follow a linear regression: $M_{\mathrm{HI}} \propto V_{\mathrm{m}} A_{25}$ (Zasov 1974). For 134 spiral galaxies Zasov \& Rubtzova (1989) derived a relation $\log M_{\mathrm{HI}} \propto(1.3 \pm 0.1) \log \left(V_{\mathrm{m}} \cdot A_{25}\right)$. Considering a sample of 535 thin (buldgeless) edge-on galaxies Karachentsev et al. (1999) found that the slope of this relation is close to unity, $k=1.08 \pm 0.03$, in the range of angular momentum covering two orders.

Figure 9 shows the relation between hydrogen mass and angular momentum for the LV galaxies. Because of the presence of a large number of small nearby galaxies, the range of their angular momentum extends over three orders. In this wide range the relation has a slope $k=0.99 \pm 0.04$, correlation coefficient $r=0.89$, and the standard deviation $\sigma\left(\log M_{\mathrm{HI}}\right)=0.36$. Therefore, we may consider that the gaseous disks of giant spiral galaxies as well as dwarf irregular ones are situated, apparently, near the threshold of gravitational instability, favouring active star formation in them.

\section{Conclusion}

To study relations between global optical and HI parameters of galaxies we used a nearly complete sample of nearby galaxies with corrected radial velocities, $V_{0}<500 \mathrm{~km} \mathrm{~s}^{-1}$ (the KKT sample). Comparing with the previous investigation undertaken by Huchtmeier \& Richter (1988), the initial KKT sample was updated by new nearby objects and also by new more homogeneous data on galaxy 
distances, apparent magnitudes and other observational parameters. A survey of basic relations between the global characteristics of nearby galaxies is presented in Table 1 . We note the following properties among them:

1) In the whole range of diameters from $1 \mathrm{Kpc}$ to $40 \mathrm{Kpc}$ the LV galaxies follow the Tully-Fisher relation, $\log A_{25} \propto(0.99 \pm 0.06) \log V_{\mathrm{m}}$, whose linear character may have a deep evolutionary sense.

2) The hydrogen mass-to-luminosity and hydrogen mass-to- "total" mass ratios increase systematically from giant galaxies to dwarfs. The median value of $M_{\mathrm{HI}} / M_{25}$ is 0.25 , however, some very nearby dwarf galaxies, such as UGCA 292, having received no detailed study as yet, have $M_{\mathrm{HI}} / M_{25}=1-3$.

3) For the LV galaxies their "total" mass (inside the standard optical diameter)-to-the total blue luminosity ratios are concentrated in the range of 0.2 to $16 M_{\odot} / L_{\odot}$ with the median $3.0 M_{\odot} / L_{\odot}$. This ratio tends to decrease slightly from giant galaxies to dwarfs, which needs an explanation within the common idea of presence of large amount of Dark Matter in dwarf irregular systems.

4) Our sample galaxies differ in their mean surface brightness almost by a factor of 100 . The hydrogen massto-luminosity ratio for them shows a clear-cut increase towards the low surface brightness objects. A similar but less distinct relation is also seen for the total mass-toluminosity ratios. Both correlations may be caused by star formation processes going on in the galaxies.

5) To quantitatively estimate the local mass density around each LV galaxy, produced by its neighbours, we used the so-called "tidal index". Despite the differences of about six orders in the local densities, the ratios $M_{\mathrm{HI}} / L$ and $M_{25} / L$ do not show pronounced correlation with the tidal index similar to the HI-deficit or morphological segregation seen in rich galaxy clusters.

6) The amount of hydrogen mass in a galaxy and its angular momentum follow a nearly linear relation, $\log M_{\mathrm{HI}} \propto(0.99 \pm 0.04) \log \left(V_{\mathrm{m}} \cdot A_{25}\right)$ in the range of angular momentum which exceeds three orders. According to Zasov (1974) it means that the gaseous disks of giant, normal, and dwarf galaxies are situated near the threshould of gravitational instability favouring star formation in them.
Acknowledgements. This work is supported by INTAS-RFBR grant 95-IN-RU-1390 and DFG grant KS 9112.

\section{References}

Bothun G., Impey C., McGaugh S., 1997, PASP 109, 745

Broeils A.H., 1992, Dark and Visible Matter in Spiral Galaxies, Dissertation. Groningen

Broeils A.H., Rhee M.H., 1997, A\&A 324, 877

Carignan C., Beaulieu S., 1989, ApJ 347, 760

Giovanelli R.G., Haynes M.P., 1991, ARA\&A 29, 499

Cote S., Freeman K.C., Carignan C., Quinn P.J., 1997, AJ 114, 1313

Dressler A., 1984, ARA\&A 22, 185

Hoffman G.L., Salpeter E.E., Fathat B., Roos T., Williams H., Helou G., 1996, ApJS 105, 269

Huchtmeier W.K., Karachentsev I.D., Karachentseva V.E., 1997, A\&A 322, 375

Huchtmeier W.K., Karachentsev I.D., 1999, A\&A (in preparation)

Huchtmeier W.K., Richter O.G., 1988, A\&A 203, 237 (HR)

Karachentsev I.D., 1994, Astron. Astrophys. Trans. 6, 3

Karachentsev I.D., Tikhonov N.A., 1994, A\&A 286, 718

Karachentsev I.D., Karachentseva V.E., Kudrya Yu.N., 1999, Lett. Astron. Zh. 25, 3

Karachentsev I.D., Makarov D.I., 1996, AJ 111, 535

Karachentsev I.D., Makarov D.I., 1998, in Procccedings of IAU Symp. No 186. Kyoto, p. 109

Karachentseva V.E., Karachentsev I.D., 1998, A\&AS 127, 409

Kraan-Korteweg R.C., Tammann G.A., 1979, Astron. Nachr. 300, 181 (KKT)

Larson R.B., Tinsley B.M., 1978, ApJ 219, 46

McGaugh S.S., de Blok W.J.G., 1997, ApJ 481, 689

Paturel G., Fouque P., Bottinelli L., Gouguenheim L., 1992, Catalogue of Principal Galaxies. Lyon (PGC)

Roberts M.S., 1969, AJ 74, 859

Roberts M.S., Haynes M.P., 1994, ARA\&A 32, 115

Salpeter E.E., Hoffman G.L., 1996, ApJ 465, 595

Schlegel D.J., Finkbeiner D.P., Davis M., 1998, ApJ 500, 525

Staveley-Smith L., Davies R.D., 1988, MNRAS 231, 833

Tully R.B., 1988, Nearby Galaxy Catalog. Cambridge Univ. Press

Tully R.B., Fisher J.R., 1977, A\&A 54, 661

Zasov A.V., 1974, AZh 51, 1225

Zasov A.V., Rubtzova T.V., 1989, Lett. Astron. Zh. 15, 118 